\title{
Implementation of a Family Intervention for Individuals with Schizophrenia
}

\author{
Amy N. Cohen, $P h D^{1,2}$, Shirley M. Glynn, PhD ${ }^{2,3}$, Alison B. Hamilton, $P h D, M P H^{1,2}$, \\ and Alexander S. Young, MD, MSHS ${ }^{1,2}$
}

'VA Desert Pacific Mental Health Research, Education and Clinical Center (MIRECC), Los Angeles, CA, USA; ${ }^{2}$ Department of Psychiatry and Biobehavioral Sciences, University of California Los Angeles, Los Angeles, CA, USA; ${ }^{3}$ Greater Los Angeles VA Healthcare System at West Los Angeles, Los Angeles, CA, USA.

BACKGROUND: Families are rarely included in clinical care despite research showing that family involvement has a positive effect on individuals with schizophrenia by reducing relapse, improving work functioning, and social adjustment.

OBJECTIVES: The VA QUERI study, EQUIP (Enhancing QUality of care In Psychosis), implemented family services for this population.

DESIGN: At two VA medical centers, veterans with schizophrenia and their clinicians were interviewed separately at baseline and 15 months. A family intervention was implemented, and a process evaluation of the implementation was conducted.

PARTICIPANTS: Veterans with schizophrenia $(\mathrm{n}=173)$ and their clinicians $(n=29)$.

INTERVENTION: Consent to contact family was obtained, mailers to engage families were sent, families were prioritized as high need for family services, and staff volunteers were trained in a brief three-session family intervention.

MAIN RESULTS: Of those enrolled, 100 provided consent for family involvement. Seventy-three of the 100 were sent a mailer to engage them in care; none became involved. Clinicians were provided assessment data on their patients and notified of 50 patients needing family services. Of those 50, 6 families were already involved, 34 were never contacted, and 10 were contacted; 7 new families became involved in care. No families were referred to the family psychoeducational program.

CONCLUSIONS: Uptake of the family intervention failed due to barriers from all stakeholders. Families did not respond to the mailer, patients were concerned about privacy and burdening family, clinicians had misperceptions of family-patient contact, and organizations did not free up time or offer incentives to provide the service. If a full partnership with patients and families is to be achieved, these barriers will need to be addressed, and a family-friendly environment will need to be supported by clinicians and their organizations. Applicability to family involvement in other disorders is discussed.

KEY WORDS: mental health; family; quality improvement.

J Gen Intern Med 25(Suppl 1):32-7

DOI: $10.1007 / \mathrm{s} 11606-009-1136-0$

(c) Society of General Internal Medicine 2009 chizophrenia is the most common serious mental illness (SMI) and, when poorly treated, results in substantial morbidity and mortality. In terms of treatment, $31 \%$ of individuals with a SMI are seen for their psychiatric issues in general medical settings, and another $42 \%$ are seen in specialty settings. ${ }^{1}$ It is well established that many types of family interventions are efficacious in reducing exacerbations of schizophrenia ${ }^{2,3}$ and have beneficial effects on social relationships, work functioning, and social adjustment. ${ }^{4}$ As a result of this evidence, family services, as part of a comprehensive, effective treatment program, are included in several best practice guidelines. ${ }^{5-7}$ In 2008 the Veteran's Health Administration (VA) released the Uniform Mental Health Services Package mandating that family services be available to all veterans with SMI. ${ }^{8}$ However, neither inclusion in best practices nor mandating availability of a service guarantees that the service will be implemented successfully or utilized.

Implementation and utilization of family services by individuals with SMI both inside and outside the VA has been very limited, resulting in a significant gap between research and practice. An analysis of third-party claims from participants in the Schizophrenia Patient Outcomes Research Team (PORT) ${ }^{6}$ survey found that only $0.7 \%$ of Medicare claims and $7.1 \%$ of Medicaid claims were for family therapy. ${ }^{9}$ In an examination of care quality at a community and VA clinic, $68 \%$ of the sample reported having a close family member, yet almost $40 \%$ of these families did not have any contact with the treatment team documented in their charts. ${ }^{10}$ In sum, family services are rarely utilized as part of routine care, and the majority of families are not having regular contact with the treatment team.

Findings in implementation science broadly indicate that barriers to successful implementation of a new intervention include a top-down mandate to implement the intervention, individual resistance from line-staff, and varying opinions about the utility of the intervention by governing bodies. ${ }^{11}$ Passive approaches to disseminating new information to clinicians (e.g., distribution of literature on efficacy studies) do not typically induce behavior change, lead to implementation success, or improve care. ${ }^{12,13}$ Findings specific to the implementation of family services indicate that common obstacles include staff stress from their workload, ${ }^{9,13,14}$ cost, agency bureaucracy, and staff skepticism about the effectiveness of the intervention. ${ }^{9,13}$

Successfully adopted interventions typically include (1) early involvement of opinion leaders that can influence future, potential users; ${ }^{15}$ (2) set plans for roll-out, adoption, and 
sustaining of the new intervention; (3) personal contact between the intervention developer and adopters, especially live demonstrations, for purposes of modeling and building momentum for use; (4) ongoing contact between developer and adopters to address implementation barriers; and (5) set incentives for adoption and competent use of new intervention. ${ }^{11}$ Reports regarding family services specifically indicate that initial positive assumptions by stakeholders about family services improve chances for implementation and sustainability. ${ }^{13}$ Additionally, extended training in the family model or trainings utilizing multi-methods produce superior outcomes compared to brief trainings or those only using didactic methods. ${ }^{9,15-17}$

The VA QUERI study EQUIP (Enhancing QUality of care In Psychosis) was a randomized controlled trial comparing usual care to interventions embedded in a chronic care model. Embedded interventions included: (1) treatment assertiveness (identifying patient needs) and care coordination, (2) guidelineconcordant medication management, and (3) a family intervention. (A full description of EQUIP can be found in Brown et al. ${ }^{18}$ ) We used mixed methods to study the implementation of the family intervention with two objectives: (1) perform a process analysis of implementation and (2) examine utilization of the intervention.

\section{METHODS}

\section{Clinic Sites and Sampling}

Data were collected at specialty mental health clinics within the Greater Los Angeles and Long Beach VA medical centers starting in January 2002. All clinicians at these clinics were eligible for the study. Patients were eligible if they were (1) at least 18 years old, (2) had a diagnosis of schizophrenia or schizoaffective disorder, (3) had at least one clinic visit during a 4-month sampling period, and (4) had at least one clinic visit during a 5-month enrollment period. Visit-based sampling was designed to be representative of those in outpatient treatment for schizophrenia ${ }^{19}$; the response rate was $51 \%$. Written informed consent was obtained from each clinician and patient, or their conservator if applicable. The study had IRB approval.

\section{Assessments}

All enrolled patients completed a baseline interview providing basic demographics and information on kin relationships including frequency of contact. Diagnosis was confirmed using an abbreviated version of the Structured Clinical Interview for DSM-IV, Patient Edition, version 2.0 (SCID). The 15-month follow-up interview was similar in content to the baseline interview.

All enrolled clinicians were asked to complete a questionnaire at baseline and 15 months. The instrument gathered data on psychiatrists' training, knowledge about schizophrenia, and workload. Included in the questionnaire was the family involvement subscale from the Competency Assessment Instrument (CAI), ${ }^{20}$ which rates competency from 0 , no competency, to 1, complete competency. The Maslach Burnout Inventory (MBI): Human Services Survey ${ }^{21}$ was also included, and norms for mental health workers were utilized to determine burnout levels of staff.
All enrolled clinicians were asked to complete a semistructured interview, conducted by the study Principal Investigator (ASY) or Project Director (ANC), at baseline and 15 months. The baseline interview included two open-ended questions regarding family services: "Thinking now about the fact that we will attempt to involve, educate, and do brief family therapy with patients and their caregivers...(1) what barriers do you anticipate in this part of the care model? and (2) what factors do you see as facilitators, likely to contribute to the success of this step?" At the final interview clinicians were asked: "Thinking now about the fact that we attempted to involve, educate, and do brief family therapy with patients and their caregivers, did the care model that we implemented have an effect on family involvement in your treatment?" Detailed interview notes were recorded. Main themes were identified by consensus of the Principal Investigator and Project Director.

To more fully understand the process of implementation and facilitate an unbiased assessment, qualitative interviews with a subset of the clinicians were conducted by an independent expert in qualitative methods $(\mathrm{ABH})$ at 15 months. These interviews were more thorough, with $30 \mathrm{~min}$ focused on discussion of the family intervention. Individuals were asked open-ended questions about their perspectives on family involvement. For example, clinicians were asked, "Many patients met one or more of [the criteria] for family involvement. Were you more involved with families... as a result? Did anything stand in your way? Were there times when you felt it wouldn't be helpful to have the family involved? What were the reasons?" Detailed interview notes were recorded. Main themes were identified by consensus of the contractor and Project Director.

Referral and utilization data for the intervention were gathered from tracking kept by each site's nurse care coordinator.

\section{Implementation of the Family Intervention}

The EQUIP family intervention had two parts. The goal of Part A was to engage families in the care of their loved one. Families were sent a personalized letter from the patient's clinician (either psychiatrist or nurse) explaining the desire to increase communication, encouraging the family to call, and providing contact information. This mailer also had contact information on local National Alliance on Mental Illness (NAMI) chapters. The goal of Part B was to educate families regarding schizophrenia and its treatment. A brief three-session psychoeducational program with ongoing consultation was available to families, offered in the clinic or via phone.

For a patient's family to participate in either part of the intervention, the patient had to provide consent for family contact. To receive the mailer from the patient's clinician (Part A), the enrolled patient had to provide the name and address of a family member. To participate in the brief three-session psychoeducational program (Part B), the family had to be referred by the patient's clinician.

The Principal Investigator or Project Director met individually with each clinician to introduce study goals and the evidence base for family involvement. Both Part A and Part B of the intervention were described, and clinicians signed letters to the families of their patients for the mailer. The nurse care coordinator asked each patient for consent to contact his family. 
Throughout the 15 months of intervention, patients met with the nurse care coordinator for a brief clinical assessment immediately prior to each meeting with his psychiatrist. Assessment data were provided via a computer "pop-up" window to the patient's care team at the time of the clinical encounter. $^{22}$ Situations typically leading to poor outcomes ${ }^{23}$ were highlighted on the screen (high persisting symptoms, reports of family stress, and/or medication non-compliance). Also, the research team compiled quarterly referral lists of patients who had provided consent to contact the family, had at least weekly contact with family, and had on their most recent brief assessment reported at least one situation leading to poor outcomes.

A psychiatrist at each clinic volunteered as the opinion leader for the EQUIP intervention. Opinion leaders had weekly phone consultation with the Principal Investigator. Staff clinicians were encouraged to act on the highlighted assessment data from the brief assessment and the referral lists by involving families in care and referring families to the psychoeducational program.

The nurse care coordinator and a staff nurse from each clinic were trained to deliver the psychoeducational program. An expert in family services (SMG) led the training using modified educational materials from validated evidence-based family psychoeducational programs. ${ }^{24}$ The training was didactic supplemented with roleplays and written materials. Throughout implementation, trainees had access to phone consultation with the family expert as needed.

\section{RESULTS}

Of the 29 enrolled clinicians, 28 completed the baseline interview and survey. Of these 28,18 were psychiatry residents, 9 were staff psychiatrists, and 1 was a nurse practitioner. At follow-up, 7 residents had graduated, 3 staff refused, and 1 staff member had no current patients in the study, leaving 18 clinicians who completed the 15-month interview and survey. For the qualitative interview at 15 months, the contractor interviewed 11 individuals: 6 psychiatrists, 3 nurse care managers, and the 2 study nurse care coordinators.

A total of 173 patients completed the baseline survey, and their demographics are shown in the Table 1. Eighty percent of the patients $(n=139)$ completed the 15-month follow-up survey.

\section{Implementation Results}

Of the 173 patients randomized to the care model, 100 (58\%) provided consent to staff to speak with a family member, 32 (18.5\%) reported they did not have any family alive, 20 (11.6\%) had family but the patient refused consent, and 21 (12.1\%) patients were never asked for consent. As Figure 1 displays, of the 100 patients who provided consent to involve their family in care, $73(73 \%)$ were sent the mailer from the patient's care team. For the remaining 27 , the patient could not provide a complete address despite multiple requests from the nurse care coordinator. Following the mailer, three family members (4\%) called the clinic. As a result of those three calls from family members, no families were engaged in treatment or referred to the psychoeducational program.
Table 1. Baseline Patient Demographics

\begin{tabular}{ll}
\hline \hline Age (average; in years) & $51.8(\mathrm{SD}=9.2)$ \\
Male gender & $90.2 \%(\mathrm{n}=156)$ \\
Race & \\
Caucasian & $69.4 \%(\mathrm{n}=120)$ \\
African-American & $18.5 \%(\mathrm{n}=32)$ \\
Latino & $7.5 \%(\mathrm{n}=13)$ \\
Asian & $4.0 \%(\mathrm{n}=7)$ \\
Native American & $0.6 \%(\mathrm{n}=1)$ \\
Illness length (average; in years) & $24.8(\mathrm{SD}=11.2)$ \\
Marital status & \\
Never married & $48.6 \%(\mathrm{n}=84)$ \\
Divorced & $26.0 \%(\mathrm{n}=45)$ \\
Currently married/living together & $13.9 \%(\mathrm{n}=24)$ \\
Separated & $7.5 \%(\mathrm{n}=13)$ \\
Widowed & $4.0 \%(\mathrm{n}=7)$ \\
\hline
\end{tabular}

At each clinical encounter with an enrolled patient, clinicians received the brief assessment "pop-up" with situations typically leading to poor outcomes highlighted. Additionally, the research team identified 50 (50.0\%) of the 100 patients who had provided consent for family contact, who had at least weekly family contact and reported at least one of the situations leading to poor outcomes. Of these 50 individuals identified for family involvement, 27 (54\%) were identified at more than one time point over the course of the study. Of the 50 referrals, $6(12 \%)$ were already receiving family and/or marital therapy at the VA at the time of the referral. Of the remaining 44 referrals, only 10 (23\%) were contacted by staff. These contacts led to seven families becoming engaged and involved in the treatment of their loved one, but no referrals to the psychoeducational program. The remaining 34 referrals $(77 \%)$ were not contacted by staff for family intervention. Several clinicians mentioned, during the 15-month semi-structured interview, that the family intervention did get professional caregivers at supervised residences more involved with the clinical team, although they were not a direct target of this intervention.

\section{Evaluation of barriers and facilitators}

Clinicians. Clinician competency (knowledge, skills, attitudes) in family involvement was $0.49(\mathrm{SD}=0.22)$ at baseline, indicating a moderate level of competency that was similar at 15 months $(0.44, \mathrm{SD}=0.19)$. When clinicians self-reported on including families in their practice in the month prior to baseline, 22 (79\%) reported teaching family members about mental illness at least weekly, 21 (74\%) reported gathering information from family members or friends at least weekly, and $20(71 \%)$ reported helping family members cope with stress at least weekly.

No clinician had MBI scores indicating "job burnout" at baseline or 15 months. The MBI defines job burnout as high scores on the subscales of emotional exhaustion (EE) and depersonalization (D) in addition to a low score on personal accomplishment (PA). The MBI subscales did indicate that $10(37 \%)$ clinicians reported high levels of EE. Ten more reported high levels of $\mathrm{D}$ at baseline, while 23 (82\%) reported low PA in their job. These findings were similar at 15 months (35\% high EE, 47\% high D, 94\% low PA). No respondent reported a high level of personal accomplishment at either time point. 


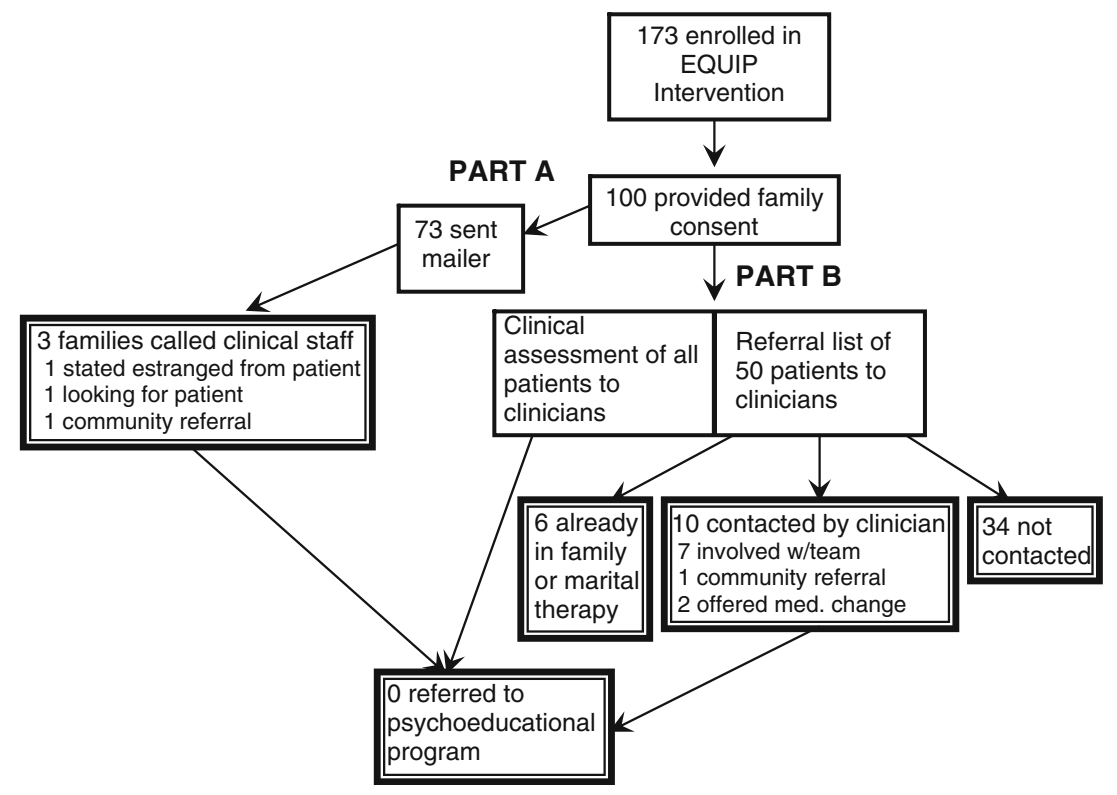

Figure 1. EQUIP family intervention results.

At baseline, the majority of clinicians responded similarly to the open-ended question about anticipated barriers to implementation of the family intervention. These responses fell into two categories: a lack of eligible individuals because "most patients do not have supportive families" and clinician time constraints. The fact that many clinicians identified similar barriers without prompting lends credence to the salience of the findings. One clinic had case managers; their presence was reported by several providers as an expected facilitator to engaging families.

At the 15-month semi-structured interview, clinicians were asked if the family intervention had any effect. Many responses matched the anticipated barrier that patients either do not have family or are estranged from family; this barrier was also described in the independent qualitative interview, with respondents noting that many patients have "burned bridges" with their families. In general, families that were accessible were seen as "dysfunctional," "inconsistent," and "usually the problem," with supportive families being the exception. However, all respondents expressed willingness to work with families, and all reported having made referrals to NAMI groups.

Patients and Families. Of the 100 patients who provided consent to talk to their families, $81(81 \%)$ had reported having a supportive family member. Of these 81 , the majority either lived with that family member $(n=40)$ or lived near that family member $(n=29)$. Of the 41 who did not live with their supportive family, many (26 or $63 \%$ ) had at least weekly contact. The majority of supportive family members (44 or $54 \%$ ) had either never had contact with the patient's care team or had no contact in the previous year; a minority (13 or 16\%) had had contact in the previous month.

Of the same 100 patients, 88 completed the 15-month survey. In response to the question, "Would you like your [supportive family] involved (or more involved) with your treatment providers here at the mental health clinic?" 77
(88\%) responded "no;" their top concerns were privacy (20 or $26 \%$ ), overburdened family (11 or $14 \%$ ), and geographical distance from family ( 11 or $14 \%$ ). Of the 11 who said yes, the main services desired were for family-psychiatrist meetings $(73 \%, n=8)$, family-case manager meetings $(36 \%, n=4)$, and regular family counseling $(36 \%, n=4)$.

Organizational Issues. Although organizational issues were not a focus of the baseline or 15-month semi-structured interviews with clinicians, it was often cited during the 15-month independent qualitative interviews. In these interviews, all psychiatrists reported time pressure from other responsibilities that limited or eliminated any chance of providing family services; psychiatrists made statements such as, "the problem is resource management," there was "no time to meet with families," and "there is a waiting list for [medication management] appointments of a couple months." Some psychiatrists suggested nurse case managers would be better suited for implementing family services; nurse case managers did seem inclined to want to provide these services. One nurse case manager said she could do "45-min sessions with a family" and that she finds working with families "refreshing," and another said "I could do family sessions but I would want more training." And while one nurse case manager said "the VA is very supportive of family involvement," other case managers said the VA "does not address evenings and babysitters [for families with children]."

\section{DISCUSSION}

Although efficacy studies indicate positive effects of family interventions when a loved one has schizophrenia, studies examining the implementation and utilization of such programs in routine care are limited. This study includes a random, representative sample of patients in care for schizo- 
phrenia and their clinicians across two VA mental health clinics. The patient sample was mostly older, unemployed, and single. Clinicians were a mix of staff psychiatrists, residents, and nurse case managers who each had at least 1/ 3 of their caseloads comprised of patients with psychosis.

The failed uptake of the family intervention suggests that there were many obstacles to its use. Clinicians reported high levels of contact with family prior to the study, but given the reports of infrequent contact reported by patients, it is likely that this was a very small minority of the pool of possible families. Clinicians' competency in getting families involved in care was considered to be moderate and higher than that reported in a sample of managed care clinicians from the community, ${ }^{20}$ but there was little follow-through with families of patients identified as possibly benefitting from family services. This lack of follow-through could be due in part to clinicians' perceptions that patients did not have supportive and/or available family members.

Contrary to these perceptions, however, many patients reported having supportive families, being in contact with their families, and patients readily provided consent for family contact when asked. It may be, though, that patients did not encourage their families to become involved given the high rate $(88 \%)$ of patients who reported at the final interview that they did not want their families involved in their care. It is possible that patients distinguished between "providing consent to contact" and "having family involved," with the latter representing an intensity of involvement that was not desired.

Organizationally, the participating VA clinics lacked the resources and supports necessary to function as familyfriendly environments including evening appointments, designated family therapists, protected appointment times for families, and performance measures regarding family involvement. ${ }^{25}$ There are considerable efforts underway to implement the VHA Uniform Mental Health Services Package, ${ }^{8}$ which may facilitate a transformation to more family-friendly clinics.

Our program implementation strategies were consistent with much of the existing evidence for successful implementation, including early involvement of opinion leaders, more thorough training and supervision, and personal contact between the intervention developer and adopters. Nevertheless, it is clear that other obstacles needed to be addressed, including staff skepticism about the benefit/risk ratio of family involvement in patient care, limited leadership support, and no reorganization of staff time, as well as few incentives for providing this clinical service. Staff's low sense of personal accomplishment and to a lesser degree emotional exhaustion and depersonalization on the job seem to be particularly problematic and warrant further attention.

The VA mental health staff in these clinics serves a predominately aging, chronic but stable, low economic resource population. Many staff may have become accustomed to seeing avoidance of crises as the primary treatment goal, and the value of newer, recovery-oriented interventions may be perceived to have little likelihood of payoff. Addressing issues of staff motivation and low levels of personal achievement as well as more rigorously employing the knowledge and tools from implementation science will be needed if a full partnership with patients and families is to be achieved.

Similar to work in schizophrenia, there is now a growing literature on the efficacy of interventions for families of persons with traumatic brain injury, ${ }^{26}$ depression, ${ }^{27}$ and dementia. ${ }^{28,29}$ While these programs can both reduce caregiver burden and improve patient functioning in controlled research settings, our results suggest that disseminating these family interventions to routine care may require overcoming many obstacles in non-specialized heath care settings where there are few incentives for medical professionals to change practices. Our results also suggest that complementary efforts must be spent both learning new interventions and developing incentive programs to assure clinicians implement the new techniques.

While implementing intensive family interventions programs may be difficult, even more modest programs aimed at involving families with the care team have been underdeveloped in the literature. For example, in recent studies on collaborative care models of depression, even though mention is made of involving families in care, ${ }^{30}$ to date no systematic reports have been made of optimal ways to involve families as part of the collaborative care model. Only recently has there been qualitative work examining the needs of families in poststroke care, ${ }^{31}$ which grew out of low attendance rates and equivocal efficacy studies in this population. ${ }^{32,33} \mathrm{~A}$ recent positive trial of collaborative care in dementia did include caregivers, ${ }^{34}$ but again it remains to be seen whether such a program can be successfully disseminated to non-research settings.

Interventions for family members of individuals with serious psychiatric or physical illnesses have been found to be efficacious and are included in numerous illness best practices, but without attention to implementation these services are often minimally utilized and not sustained. This can lead to assumptions that the service is not needed or is ineffective, when in reality it was never successfully implemented. Implementation science is a burgeoning field that offers data that can direct plans for roll-out, uptake, and sustaining an intervention, such as family services. Our work indicates that for family services to be utilized, additional attention needs to be paid to reorganization of care practices, clinician competencies, and education to patients and families regarding their role in the patient's recovery and rehabilitation.

Acknowledgements: We would like to acknowledge the contributions of Michelle Briggs, RN, Daniel Mezzacapo, RN (deceased), Kirk McNagny, MD, and Christopher Kessler, MD. An earlier draft of this work was presented as a poster at the VA Health Services Research and Development National Conference in February 2008 and as a paper at the VA Mental Health National Conference in July 2008. This material is based upon work supported by the Department of Veterans Affairs, Veterans Health Administration, Office of Research and Development, Health Services Research and Development (RCD 00-033 and CPI 99-383) and the Desert Pacific Mental Illness Research, Education and Clinical Center (MIRECC), and by the UCLA-RAND NIMH Partnered Research Center for Quality Care (MH068639 and MH082760). The views expressed in this article are those of the authors and do not necessarily reflect the position or policy of the Department of Veterans Affairs or the United States government.

Conflict of Interest: None disclosed.

Corresponding Author: Amy N. Cohen, PhD; Department of Psychiatry and Biobehavioral Sciences, University of California Los Angeles, Los Angeles, CA, USA (e-mail: amy.cohen@va.gou). 


\section{REFERENCES}

1. Narrow W, Regier D, Norquist G, Rae D, Kennedy C, Arons B. Menta health service use by Americans with severe mental illnesses. Soc Psychiatry Psychiatr Epidemiol. 2000;35:147-55.

2. Pilling S, Bebbington $\mathbf{P}$, Kuipers $\mathbf{E}$, et al. Psychological treatments in schizophrenia: I. Meta-analysis of family intervention and cognitive behaviour therapy. Psychol Med. 2002;32:763-82.

3. Pitschel-Walz G, Leucht S, Bauml J, Kissling W, Engel R. The effect of family interventions on relapse and rehospitalization in schizophrenia-a meta-analysis. Schizophr Bull. 2001;27:73-92.

4. Chien w, Chan S. One-year follow-up of a multiple-family-group intervention for Chinese families of patients with schizophrenia. Psychiatr Serv. 2004;55:1276-84.

5. American Psychiatric Association. Practice Guideline for the Treatment of Patients With Schizophrenia. 2nd ed. Arlington, VA; 2004.

6. Lehman A, Kreyenbuhl J, Buchanan $\mathbf{R}$, et al. The Schizophrenia Patient Outcomes Research Team (PORT): updated treatment recommendations 2003. Schizophr Bull. 2004;30:193-217.

7. McEvoy J, Scheifler P, Frances A. The expert consensus guideline series: treatment of schizophrenia 1999. J Clin Psychiatry. 1999;60

8. Department of Veterans Affairs. Uniform mental health services in VA medical centers and clinics 2008. VA Handbook 1160.01 .

9. Dixon L, Lyles A, Scott $\mathbf{J}$, et al. Services to families of adults with schizophrenia: from treatment recommendations to dissemination. Psychiatr Serv. 1999;50:233-8.

10. Young AS, Sullivan G, Burnam MA, Brook RH. Measuring the quality of outpatient treatment for schizophrenia. Arch Gen Psychiatry. 1998;55:611-7.

11. Backer T, Liberman R, Kuehnel T. Dissemination and adoption of innovative psychosocial interventions. J Consult Clin Psychol. 1986;54:111-8.

12. Gilbody S, Whitty $\mathbf{P}$, Grimshaw $\mathbf{J}$, Thomas $\mathbf{R}$. Educational and organizational interventions to improve the management of depression in primary care: a systematic review. J Am Med Assoc. 2003;289:3145-51.

13. McFarlane W, McNary S, Dixon L, Hornby H, Cimett E. Predictors of dissemination of family psychoeducation in community mental health centers in Maine and Illinois. Psychiatr Serv. 2001;52:935-42.

14. Barrowclough $\mathbf{C}$, Tarrier $\mathbf{N}$, Lewis $\mathbf{S}$, et al. Randomised controlled effectiveness trial of a needs-based psychosocial intervention service for carers of people with schizophrenia. Br J Psychiatry. 1999;174:505-11.

15. Amenson C, Liberman R. Rehab rounds: dissemination of educationa classes for families of adults with schizophrenia. Psychiatr Serv. 2001;52:589-92.

16. Farhall J, Webster B, Hocking B, Leggatt M, Riess C, Young J. Training to enhance partnerships between mental health professionals and family caregivers: a comparative study. Psychiatr Serv. 1998;49:1488-90.

17. Mairs H, Bradshaw T. Implementing family intervention following training: what can the matter be? J Psychiatr Ment Health Nurs. 2005; 12:488-94.
18. Brown AH, Cohen AN, Chinman MJ, Kessler C, Young AS. EQUIP: implementing chronic care principles and applying formative evaluation methods to improve care for schizophrenia: QUERI Series. Implement Sci. 2008;3:9

19. Young A, Sullivan G, Burnam M, Brook R. Measuring the quality of outpatient treatment for schizophrenia. Arch Gen Psychiatry. 1998;55:611-7

20. Chinman M, Young A, Rowe M, Forquer S, Knight E, Miller A. An instrument to assess competencies of providers treating severe mental illness. Ment Health Serv Res. 2003;5:97-108.

21. Maslach C, Jackson S, Leiter M. Maslach burn out inventory manual, 3rd ed. Palo Alto, CA: Consulting Psychologists Press; 1996.

22. Young A, Mintz J, Cohen A, Chinman M. A network-based system to improve care for schizophrenia: the Medical Informatics Network Tool (MINT). J Am Med Inform Assoc. 2004;11:358-67.

23. Leff J. Family work for schizophrenia: practical application. Acta Psychiatr Scand Suppl. 2000:78-82.

24. Mueser K, Glynn S. Behavioral family therapy for psychiatric disorders, 2nd ed. Oakland, CA: New Harbinger Publications, Inc.; 1999.

25. Cohen AN, Glynn SM, Murray-Swank AB, et al. The family forum: directions for the implementation of family psychoeducation for severe mental illness. Psychiatr Serv. 2008;59:40-8.

26. Rivera PA, Elliott TR, Berry JW, Grant JS. Problem-solving training for family caregivers of persons with traumatic brain injuries: a randomized controlled trial. Arch Phys Med Rehabil. 2008;89:931-41.

27. Jacobson NS, Dobson K, Fruzzetti AE, Schmaling KB, Salusky S. Marital therapy as a treatment for depression. J Consult Clin Psychol. 1991;59:547-57.

28. Gitlin LN, Belle SH, Burgio LD, et al. Effect of multicomponent interventions on caregiver burden and depression: the REACH multisite initiative at 6-month follow-up. Psychol Aging. 2003;18:361-74.

29. Gitlin LN, Winter L, Corcoran M, Dennis MP, Schinfeld S, Hauck WW. Effects of the home environmental skill-building program on the caregiver-care recipient dyad: 6-month outcomes from the Philadelphia REACH Initiative. Gerontologist. 2003;43:532-46.

30. Katon W, Von Korff M, Lin E, et al. Stepped collaborative care for primary care patients with persistent symptoms of depression: a randomized trial. Arch Gen Psychiatry. 1999;56:1109-15.

31. White CL, Korner-Bitensky $\mathbf{N}$, Rodrigue $\mathbf{N}$, et al. Barriers and facilitators to caring for individuals with stroke in the community: the family's experience. Can J Neurosci Nurs. 2007;29:5-12.

32. Korner-Bitensky N, Tarasuk J, Nelles J, Michallet B. The impact of interventions with families post-stroke: a review. Top Stroke Rehabil. 1998:69-85.

33. Rodgers H, Atkinson C, Bond S, Suddes M, Dobson R, Curless R. Randomized controlled trial of a comprehensive stroke education program for patients and caregivers. Stroke. 1999;30:2585-91.

34. Callahan CM, Boustani MA, Unverzagt FW, et al. Effectiveness of collaborative care for older adults with Alzheimer disease in primary care: a randomized controlled trial. JAMA. 2006;295:2148-57. 